\title{
Aberrations in Theories of Optical Aberrations
}

\author{
*P. Rama Krishna ${ }^{1}$ and D. Karuna Sagar ${ }^{2}$ \\ ${ }^{1}$ Asst. Prof. of Physics, Government Degree College, Armoor- 503 224, India \\ ${ }^{2}$ Professor, Department of Physics, Osmania University, Hyderabad -500 007, India
}

\begin{abstract}
Optical imaging systems find applications from medicine to military, nano chip to deep space. Their imaging efficiency is deteriorated by Aberrations. Aberration theory has very long history of development. Different types of aberrations, different notations of their description are available in the literature. Often a situation of ambiguity arises for an interdisciplinary scholar to understand them. This paper emphasizes the need of commonness and presents an effort to put them at one place.
\end{abstract}

Keywords: Optical imaging, Aberrations, Zernike polynomials, ENZ theory

\section{Introduction}

Optical imaging systems find diversified applications in the field of surveillance, astronomy, medicine, industry, research and development etc. Modern day electronic gadgets, imaging drones, non-invasive surgery, lithography, airborne and deep space imaging involve optical imaging devices. For an end user it may appear that they work with very high perfection or ideality. Because of the inherent nature of optical systems, the images formed are not perfect as optical aberrations degrade them. Aberrations are the perturbations in ideal image formation.

Any deviation from the actual shape and position of an image as estimated by Gaussian optics is called as an aberration, or simply aberrations are defined as departures from ideal behavior. Aberration leads to blurring of the image produced by an image-forming optical system. Learning how aberrations are computed, balanced, and corrected provides an insight into working of a lens scheme. Assessing its tolerance sensitivity, manufacturability, and other sensitive parameters guide subsequent improvement. To obtain sharp images the aberrations must be corrected, balanced, minimized or avoided. Makers of optical instruments need to correct optical systems to compensate for aberration. Aberration theory can augment numerical optimization and gives the designer a much firmer grasp of a lens system's limitations and potential [1,2,3].

Advent of new technology and better resolution of imaging are growing hand in hand. The utmost priority of this day is resolution of finer details without aberrations. To meet the demands of industry and consumer world, it is necessary to understand the aberrations and minimize them. From Seidel to modern day researchers aberration theory expanded multifold and many newer aberrations like polarization aberrations, random aberrations are included. Various fields of their application use different notation and definitions, and interpreted independently.

Ideal images are often defined by application dependent requirements. For example, lithographic systems require different set of definition linked to instrumental capabilities to assess the full field optical image quality. The performance of stepper's optical system can be judged by its ability to accurately and routinely place an image within photoresist medium. Lens aberrations can influence the exact placement and integrity of the image [4].

Optical imaging is also making substantial impact on basic and translation medical research. Optical techniques such as bioluminescence and fluorescence are emerging as powerful new 
modalities for molecular imaging in disease and therapy. LASIK surgery is very common now a day. Imaging science is crossing the boundaries of disciplines. For an interdisciplinary scholar coma brings coma. Ophthalmologist reports coma as higher order aberration, where as astrophysicist says it as either primary aberration or third order ray aberration or fourth order wave aberration depending upon context.

In addition to their type, aberrations are often specified according to their order (third-order, fifth-order, etc.), though they are called primary, secondary etc sometimes. Moreover higher order aberrations introduce new types. For example, fifth-order aberrations add the types oblique spherical aberration and elliptical coma[5]. In ophthalmic sense, aberrations are mentioned differently as Low order aberrations (LOA) and higher order aberrations (HOA) [6]. OSA made an attempt to standardize reporting of optical aberrations of eyes [7]. No such attempt is noticed in other fields of application. For a novice scholar aberration theory in available literature appears to be aberrated. An attempt is made in this paper to put all those things concerned with aberrations at one juncture.

\section{Theory and Discussion}

The phenomenon of aberrations in mirrors has been known since the distant past. Spherical aberration in spherical mirrors was pointed out by Roger Bacon in 13th century. In the 17th century Kepler and Descartes have noticed aberrations for refracting systems also. Optical aberrations, particularly spherical aberration, are studied by Newton. In spite of brilliant work done by Huygens, Smith, Clairant, Hertzberger, Euler \& Lagranges in the field of geometrical optics, a systematic study was made only after the discovery of periscopic lens and photography in early 19th century. Earlier to this objectives of the telescopes were of small aperture and field of view. The main effort in the understanding of aberrations was given to the correction of spherical aberration and chromatic change of focus. Off-axis aberrations did not get due attention because of small field of view of then available telescopes and microscopes. The theory was further developed by Seidel, Airy, Coddington, Petzveal, Abbe, Gullstrand, Schwartzchild etc. [8].

Aberration theory is studied in two parts namely, geometrical theory and wave theory. Terms involving off axis distances in power greater than two in the expansion of characteristic function represent geometrical aberrations. As the wavefronts form the orthogonal trajectories of the light rays, lack of homocentricity of the image forming light rays is accompanied by aspheric nature of wavefronts. The inseparable relation between aspheric nature of wavefront and non-homocentricity of rays establishes connection between geometrical and wave theories of aberrations.

In 1833 based on fundamental principles, Hamilton [9] introduced a function

$$
\mathrm{T}=\mathrm{T}^{(0)}+\mathrm{T}^{(2)}+\mathrm{T}^{(4)}
$$

describing the image formation in optical instruments, which establishes for the first time aberrations by order. Here, the second order term

$$
\mathrm{T}^{(2)}=\mathrm{P}_{1}\left(\alpha^{2}+\beta^{2}\right)+\mathrm{P}_{2}\left(\alpha \alpha^{\prime}+\beta \beta^{\prime}\right)+\mathrm{P}_{2}\left(\alpha^{, 2}+\beta^{, 2}\right)
$$

defines focal length $\left(\mathrm{P}_{1}\right)$, magnification $\left(\mathrm{P}_{2}\right)$ and chromatic aberration $\left(\mathrm{P}_{3}\right)$. The variables $\left(\alpha^{2}+\beta^{2}\right)$, $\left(\alpha \alpha^{\prime}+\beta \beta^{\prime}\right)$ and $\left(\alpha^{\prime 2}+\beta^{\prime 2}\right)$ are the rotational invariants. The fourth order term defines spherical and other aberrations. 
$\mathrm{T}^{(4)}=\mathrm{Q}_{1}\left(\alpha^{2}+\beta^{2}\right)^{2}+\mathrm{Q}_{2}\left(\alpha^{2}+\beta^{2}\right)\left(\alpha \alpha^{\prime}+\beta \beta^{\prime}\right)+\mathrm{Q}_{3}\left(\alpha^{2}+\beta^{2}\right)\left(\alpha^{\prime 2}+\beta^{\prime 2}\right)+\mathrm{Q}_{4}\left(\alpha \alpha^{\prime}+\beta \beta^{\prime}\right)^{2}+\mathrm{Q}_{5}\left(\alpha \alpha^{\prime}+\beta \beta^{\prime}\right)\left(\alpha^{\prime 2}+\beta^{\prime 2}\right)+\mathrm{Q}_{6}\left(\alpha^{\prime 2}\right.$ $\left.+\beta^{2}\right)^{2}$

This equation opened discussion for other possible aberrations, which were not identified till that date. In 1836 Gauss developed first order theory of image formation introducing the concept of cardinal points and planes popularly known as Gaussian optics. It was the first theoretical model describing the image formation in ideal and symmetric optical system.

In 1856 Seidel took into account all the terms of the third order in a general centered system of spherical surfaces [10]. The Seidel aberrations account for the monochromatic geometrical aberrations of centered optical systems, i.e. defects from perfect imagery in optical systems that have an optical axis. The types of aberrations can be developed from considerations of symmetry, and are given names such as spherical aberration, coma, astigmatism, field curvature, and distortion. Seidel's third order aberrations not only gave insight into the nature of different types of aberration, but also provided a powerful tool for optical system design by studying the contributions of each optical surface to the total of the aberrations, interestingly much beyond the expectations of Seidel.

It has been known for a long time that 3rd order aberration terms alone are insufficient to describe the performance of optical systems that must meet high demands in aperture and/or field, and an extension to higher orders appears to be a logical choice. Unfortunately, there are two drawbacks. First, an extension of Seidel's method to fifth order is quite cumbersome. Several attempts by different people have been recorded to overcome this hurdle. Second, the higher-order aberration coefficients suffer from a lack of correlation with the properties of the individual optical surfaces of a system.

In 1905 Schwarzschild introduced the concept of Eikonal or a characteristic function of four variables and could calculate 5th order coefficients [11]. It is helpful in understanding the propagation of light through optical system at a higher level of detail. The theory is further developed by Steward[12], Herzberger [13], Kohlschutter[14]. Aberrations of order greater than five are studied by Velzel and Meijere[15], Bociort, Anderson and Beckmann[16], but did not attract much attention owing to their less practical importance. Increase in number of aberration types and their order lead to more confusion in interpretation. This was overcome by developing aberration polynomial like Zernikes.

The magnitude of the aberrations is expressed in terms of an aberration polynomial. The aberration polynomial coefficients can be found from a series expansion of the law of refraction. Buchdahl [17] introduced an iteration procedure for rotational symmetric system to compute higherorder coefficients from low-order coefficients. Transverse ray displacements, longitudinal ray displacements, or wavefronts can be constructed using it.

Geometrical theory of aberrations will be correct only if the magnitude of aberrations is more than that of wavelength of light. In most of the instruments such large aberrations will be corrected and tolerance for small possible aberrations is checked so that the system remains useful. Keeping this in mind smaller wavefront errors are investigated at each and every point in the image plane taking phase relations into consideration.

In the mid of $20^{\text {th }}$ century Hopkins developed wave theory of aberrations based on Fourier method. His student Shack further developed it by writing aberration as function of field $(\mathrm{H})$ and aperture $(\rho)$ vectors. To precisely determine image quality it is necessary to have knowledge of the optical field at the exit pupil of an optical system, and then perform a diffraction integral. According to Kirchhoff-Fresnel formula geometrical field $\mathrm{G}(\mathrm{H}, \rho)$ at the exit pupil of an optical system is given by 


$$
G(H, \rho)=f(\rho) \cdot \exp \left\{-i \frac{2 \pi}{\lambda}(S(H, \rho)-W(H, \rho))\right\}
$$

$f(\rho)$ being the aperture function. The aberration function $\mathrm{W}(\mathrm{H}, \rho)$ is the optical path difference between sphere function $\mathrm{S}(\mathrm{H}, \rho)$ and actual wavefront. To obtain the image diffraction calculation is performed. According to Hopkins the wave aberration function is given by

$$
\mathrm{W}(\mathrm{H}, \rho)=\sum \mathrm{C}_{\mathrm{ABC}} \mathrm{h}^{\mathrm{A}} \rho^{\mathrm{B}} \cos ^{\mathrm{C}} \phi
$$

Where $\mathrm{C}_{\mathrm{ABC}}$ is the numerical constant, $\mathrm{h}$ is the image height, $(\rho, \phi)$ are the polar coordinates of ray intersection with aperture and $\mathrm{A}, \mathrm{B}, \mathrm{C}$ are the integers. $(\mathrm{A}+\mathrm{B}-1)$ is the order of the ray/geometrical aberration. Hopkin's way of representing aberrations is shown in Table -1. The relation between ray (TRA) and wave aberrations is given by

$$
T R A=-\frac{\mathrm{R}}{\mathrm{n}} \nabla \mathrm{W}
$$

Here $\mathrm{R}$ is the radius of wavefront and $\mathrm{n}$ is the index of refraction of final medium

There are 2 first order terms, 5 third order terms, and 9 fifth order terms and so on. The number of terms in $\mathrm{N}^{\text {th }}$ order is given by $2+3+4+5+\ldots \ldots \ldots+0.5(\mathrm{~N}+3)$.

The equations needed to calculate aberration coefficients are complicated, but can be readily evaluated numerically by optical design softwares. Another landmark in aberration theory is introducing Zernike polynomials. The Zernike polynomials were introduced by Frits Zernike in the early 20th century and later developed by several workers to describe the diffraction theory of aberrations [18]. Using Zernike polynomials, Nijboer represented the complex field in the exit pupil and focal plane. Like the Seidel aberration polynomial, Zernike polynomials describe defects from perfect imagery, but the nature of the Zernike expansion is different from the Seidel expansion. The Zernike coefficients are ordinarily found using a least-squares fit to a grid of exact ray data, while the Seidel coefficients can be computed from paraxial ray data. In the early days, the computational cost of Zernike coefficients severely limited their use in optical design, but the availability of low-cost and high-speed computers today has rekindled interest in the use of Zernike coefficients both to specify aspheric surfaces, and to build error functions. The beauty of Zernike polynomials is automatic balancing of aberrations of various orders. Zernike way of representing aberrations is shown in Table -2 .

Zernike polynomials describe the properties of an aberrated wavefront without regard to the symmetry properties of the system that gave rise to the wavefront. The other advantages being they form a complete set, can be readily separated into radial and angular functions, and the individual polynomials are orthogonal over the complete unit circle [19]. However, they are not orthogonal over portions of the unit circle. If the polynomials are generated by fitting to a limited set of data points, the orthogonality relationships may not be maintained. One fairly new application of Zernike polynomials in optical design is for the representation of aspheric surfaces, either refractive or diffractive. Here, the orthogonality of the coefficients is useful for introducing higher-order terms that are independent of the lower-order terms.

The common misconception is that the low-order Zernike polynomials have a one-to-one correspondence to the common Seidel aberrations. For example, the Zernike coefficient for spherical aberration $\left(Z_{4}^{-4}\right)$ is compared with the primary spherical aberration (SA3). This is misleading, because it is neither possible to compute $Z_{4}^{-4}$ from SA3, nor vice versa. If the aberrations function having no aberrations higher than third order describing a system over the entire unit circle, then such 
a correspondence may be possible. But if fifth or higher-order aberrations are present, then the equality of the third-order relationships is not maintained [20].

Nijboer extended his results for low order, small amplitude aberrations to small volumes out of focal plane. The process adopted was crude and hence could not be extended for large volumes. Braat, Dirksen and Janssen have successfully explained analytically the focal field over a large volume taking vector diffraction effects into consideration [21], and the theory is called as Extended Nijboer-Zernike(ENZ) theory. This theory is advantageous in aberration retrieval, high NA imaging, imaging of extended objects in a stratified medium.

Polarization aberrations are the changes in the phase, amplitude and polarization states of optical field across the exit pupil. Polarization effects are observable when the field in the exit pupil is taken in vector form. Diattenuation and Retardance are the two polarization effects. Polarization change also occurs at each reflecting and refracting surface due to differences between the $\mathrm{s}$ and $\mathrm{p}$ components of the light's reflectance and transmission coefficients. These polarization aberrations introduce phase changes in the diffraction image proportional to first and second derivatives of unpolarized aberrated image structure [22]. Two orthogonal fields $R_{n}$ and $T_{n}$ form an adequate basis to express the amplitude A of the optical field. These two fields are related to wave aberration function as

$$
\begin{aligned}
& \overrightarrow{R_{n}}=\vec{\nabla} W(\vec{\imath}, \vec{H}, \vec{\rho})=\vec{x} \frac{\partial W}{\partial x}+\vec{y} \frac{\partial W}{\partial y} \\
& \overrightarrow{T_{n}}=\vec{\nabla} X W(\vec{\imath}, \vec{H}, \vec{\rho})=\vec{x} \frac{\partial W}{\partial y}-\vec{y} \frac{\partial W}{\partial x}
\end{aligned}
$$

The aberration function in terms of vectors is given by

$$
W(\vec{l}, \vec{H}, \vec{\rho})=\sum_{k, m, n, p, q}^{\infty} W_{\substack{2 k+n+p, 2 m+n+q, n, p, q}}(\vec{H} \cdot \vec{H})^{k}(\vec{\rho} \cdot \vec{\rho})^{m}(\vec{H} \cdot \vec{\rho})^{n}(\vec{l} \cdot \vec{H})^{p}(\vec{l} \cdot \vec{\rho})^{q}
$$

Like Seidel and Zernike aberration terms, here also we find terms of different order. We get three first order and fifteen third order terms as a function of symmetry, field and aperture vectors.

\begin{tabular}{|c|c|c|c|c|}
\hline S.No. & Aberration Term & Name & Order & Classification \\
\hline 1 & $\mathrm{C}_{020} \rho^{2}$ & Longitudinal shift of image & 1 & \\
\hline 2 & $\mathrm{C}_{111} \mathrm{~h} \rho \cos \phi$ & Vertical shift of image & 1 & \\
\hline 3 & $C_{040} \rho^{4}$ & Spherical aberration & 3 & \multirow{5}{*}{$\begin{array}{c}\text { Seidel } \\
\text { Aberrations }\end{array}$} \\
\hline 4 & $C_{131} h \rho^{3} \cos \phi$ & Coma & 3 & \\
\hline 5 & $C_{220} h^{2} \rho^{2}$ & Petzual curvature & 3 & \\
\hline 6 & $C_{222} h^{2} \rho^{2} \cos ^{2} \phi$ & Astigmatism & 3 & \\
\hline 7 & $\mathrm{C}_{311} \mathrm{~h}^{3} \rho^{1} \cos \phi$ & Distortion & 3 & \\
\hline 8 & $C_{060} \rho^{6}$ & Spherical aberration & 5 & \multirow{4}{*}{$\begin{array}{l}\text { Schwarzschild } \\
\text { Aberrations }\end{array}$} \\
\hline 9 & $\mathrm{C}_{151} \mathrm{~h} \rho^{5} \cos \phi$ & Linear Coma & 5 & \\
\hline 10 & $\mathrm{C}_{331} \mathrm{~h}^{3} \rho^{3} \cos \phi$ & Elliptical Coma (cubic) & 5 & \\
\hline 11 & $C_{333} h^{3} \rho^{3} \cos ^{3} \phi$ & Elliptical Coma (line) & 5 & \\
\hline
\end{tabular}

\section{Tables}

Table 1: Hopkin's way of representing aberrations 


\begin{tabular}{|c|c|c|c|c|}
\hline 12 & $C_{240} h^{2} \rho^{4}$ & Oblique spherical (sagittal) & 5 & \\
\hline 13 & $C_{242} h^{2} \rho^{4} \cos ^{2} \phi$ & Oblique spherical(tangential) & 5 & \\
\hline 14 & $C_{420} h^{4} \rho^{2}$ & Petzual curvature & 5 & \\
\hline 15 & $\mathrm{C}_{420} \mathrm{~h}^{4} \rho^{2} \cos ^{2} \phi$ & Astigmatism & 5 & \\
\hline 16 & $C_{511} h^{5} \rho^{1} \cos \phi$ & Distortion & 5 & \\
\hline 17 & & n...... & 7 & \\
\hline
\end{tabular}

Table 2: Zernike way of representing aberrations

\begin{tabular}{|c|c|c|c|c|}
\hline S.No. & $\begin{array}{l}\text { Order } \\
\text { (n) }\end{array}$ & $\begin{array}{c}\text { Frequency } \\
\text { (m) }\end{array}$ & Aberration term & Classification \\
\hline 1 & 0 & 0 & 1 & \\
\hline 2 & 1 & -1 & $2 \rho \sin \phi$ & \\
\hline 3 & 1 & 1 & $2 \rho \cos \phi$ & \\
\hline 4 & 2 & -2 & $\sqrt{6} \rho^{2} \sin 2 \varnothing$ & \multirow{3}{*}{$\begin{array}{c}\text { Lower order } \\
\text { Aberrations } \\
\text { (LOAs) }\end{array}$} \\
\hline 5 & 2 & 0 & $\sqrt{3}\left(2 \rho^{2}-1\right)$ & \\
\hline 6 & 2 & 2 & $\sqrt{6} \rho^{2} \cos 2 \varnothing$ & \\
\hline 7 & 3 & -3 & $\sqrt{8} \rho^{3} \sin 3 \emptyset$ & \multirow{15}{*}{$\begin{array}{c}\text { Higher order } \\
\text { Aberrations } \\
\text { (HOAs) }\end{array}$} \\
\hline 8 & 3 & -1 & $\sqrt{8}\left(3 \rho^{3}-2 \rho\right) \sin \emptyset$ & \\
\hline 9 & 3 & 1 & $\sqrt{8}\left(3 \rho^{3}-2 \rho\right) \cos \varnothing$ & \\
\hline 10 & 3 & 3 & $\sqrt{8} \rho^{3} \cos 3 \emptyset$ & \\
\hline 11 & 4 & -4 & $\sqrt{10} \rho^{4} \sin 4 \varnothing$ & \\
\hline 12 & 4 & -2 & $\sqrt{10}\left(4 \rho^{4}-3 \rho^{2}\right) \sin 2 \varnothing$ & \\
\hline 13 & 4 & 0 & $\sqrt{5}\left(6 \rho^{4}-6 \rho^{2}+1\right)$ & \\
\hline 14 & 4 & 2 & $\sqrt{10}\left(4 \rho^{4}-3 \rho^{2}\right) \cos 2 \varnothing$ & \\
\hline 15 & 4 & 4 & $\sqrt{10} \rho^{4} \cos 4 \varnothing$ & \\
\hline 16 & 5 & -5 & $\sqrt{12} \rho^{5} \sin 5 \varnothing$ & \\
\hline 17 & 5 & -3 & $\sqrt{12}\left(5 \rho^{5}-4 \rho^{3}\right) \sin 3 \emptyset$ & \\
\hline 18 & 5 & -1 & $\sqrt{12}\left(10 \rho^{5}-12 \rho^{3}+3 \rho\right) \sin \emptyset$ & \\
\hline 19 & 5 & 1 & $\sqrt{12}\left(10 \rho^{5}-12 \rho^{3}+3 \rho\right) \cos \emptyset$ & \\
\hline 20 & 5 & 3 & $\sqrt{12}\left(5 \rho^{5}-4 \rho^{3}\right) \cos 3 \emptyset$ & \\
\hline 21 & 5 & 5 & $\sqrt{12} \rho^{5} \cos 5 \emptyset$ & \\
\hline
\end{tabular}

\section{Conclusion}

Imaging science finds diversified applications. Theory of aberrations is explained in different ways in different fields of application. To avoid the ambiguity in the theory for an interdisciplinary scholar a consensus in definitions, notation of aberrations must be evolved. The discussion presented in this paper brings about an oversight of different types of optical aberrations and their notation. A dearth of consensus is emphasized.

\section{Acknowledgements}

Authors thank S N Tandon, Emeritus Professor, IUCAA, Pune and S R Bharadwaj, Director, Brien Holden Institute of Optometry and Vision Sciences, LVPEI, Hyderabad for useful discussions. 


\section{References}

[1]. W. T. Welford, Aberrations of Optical Systems, (Adam Hilger, Bristol, UK, 1991)

[2]. R. Kingslake, R. B. Johnson, Opt.Photon News,5, 1994, 20-23

[3]. W. J. Smith, Modern Optical Engineering, (McGraw-Hill, New York, NY, 2007)

[4]. D. G. Flagello et. al., Theory of high-NA imaging in homogeneous thin films, JOSA A ,13,1996, 53-64

[5]. H. H. Hopkins, On the diffraction theory of optical images, Proc. R. Soc., London, Ser.A,217, 1953, 408-432

[6]. OSLO Version 5 Optics Reference, Sinclair Optics ,1997

[7]. L. N. Thibos et al., Standards for reporting the optical aberrations of eyes, J. Refra. Surg.,18,2002, 652-660

[8]. H. H. Hopkins, Wave theory of aberrations, (Oxford, Clarendon Press, 1950)

[9]. W. R. Hamilton, Trans. Irish Acad.15,69,1828; Brit. Assoc.Rep.,360,1833

[10]. L. Seidel, Astr. Nachr. 43,1856, 289-304.

[11]. K. Schwarzschild, Untersuchungen zur geometr. Optik, I, Astr. Mitt. Kgl. Sternwarte zu Gottingen, 1905

[12]. G. C. Steward, Trans. Camb. Phil. Soc. 23,1924, 235

[13]. M. Herzberger, J.Opt.Soc.Am. 29 , 1939, 395-406

[14]. Nijboer, The diffraction theory of aberrations, Ph.D.thesis, University of Groningen, 1942

[15]. Meijere, Velzel, JOSA A 6(10),1989,1609-1617

[16]. F. Bociort, T. B. Anderson, L. H. Beckmann, Appl.Opt,47(30),,2008, 5691-5700

[17]. H. A. Buchdahl, Optical Aberration Coefficients, (Dover Publications, 1968).

[18]. M. Born and E. Wolf, Principles of Optics, (Pergamon Press, London, 1959)

[19]. C. J. Kim and R. R. Shannon, Catalog of Zernike Polynomials, Applied Optics and Optical Engineering, Vol. X, Chapter 4, (Academic Press, 1987)

[20]. J. C. Wyant and Katherine Creath, Basic Wavefront Aberration Theory for Optical Metrology, Applied Optics and Optical Engineering, Vol. XI, Chapter 1, (Academic Press, 1992)

[21]. J. J. M. Braat, P. Dirksen, A. J. Janssen, JOSA A,19, 2002, 858 -870

[22]. J. B. Breckinridge, W. S. T. Lam, and R. A. Chipman, Astro. Soc. of Paci., 127,2015, 445-468

IOSR Journal of Applied Physics (IOSR-JAP) is UGC approved Journal with Sl. No. 5010, Journal no. 49054.

P. Rama Krishna. "Aberrations in Theories of Optical Aberrations." IOSR Journal of Applied Physics (IOSR-JAP) 9.4 (2017): 37-43. 